\title{
Correspondence
}

Epidemiol. Infect. (2011).

doi: $10.1017 /$ S0950268810002244

First published online 28 September 2010

\section{Smoking and other risk factors for hantavirus infections: the whole story}

To the Editor:

We read with great interest the recent case-control study by Vapalahti et al. [1] on risk factors for nephropathia epidemica (NE). In their article, the authors describe 'smoking as the most striking and clear risk factor'. This interesting finding came as no surprise, since NE is actually caused by inhalation of aerosolized PUUV particles, leading to an acute viral illness with possible lung symptoms which may be predominant, even in Europe [2]. Smoking may also increase the risk of ingestion of rodent faeces or urine through poor hand hygiene. Nevertheless, PUUV and all other pathogenic hantaviruses worldwide are almost never listed as 'respiratory viruses', although these emerging infections, and particularly the New World variants, have caused more casualties so far than two other emerging respiratory pathogens, SARS and avian influenza [3].

However, the authors omitted to mention that smoking had already been reported as a major risk factor more than 11 years ago in the earliest European case-control study on NE, completed after the first major NE peak in 1993 in Belgium [4]. In this admittedly much smaller study, 41 seroconfirmed NE cases were compared to 37 seronegative household controls and 32 seronegative neighbourhood controls. This omission is all the more remarkable, since the Belgian study is included by the Finnish authors in their list of references. Smoking and several other striking points of resemblance between their study and the Belgian study are not, however, mentioned, including similarity of the mean age of the NE cases (46 years $v s$. 44 years), absence of children $<15$ years in both studies, predominance of males in the study group but of females in the control group, use of matched pairs with seronegative controls living in the neighbourhood, a majority of cases living in a one-family house, and in a rural (often forested) area, etc. As suggested by the Finnish authors in their last paragraph, similar studies have the great merit of corroborating each other if very similar outcomes are reached, and should consequently be highlighted, rather than ignored. Indeed, smoking emerged as the second most important risk factor for NE in the Belgian study, with an odds ratio (OR) of $9 \cdot 1(95 \%$ CI $3 \cdot 2-25 \cdot 9, P=0 \cdot 01)$ for cases $v s$. controls. To rule out confounding factors for similar living conditions among family controls, a comparison was made with neighbouring controls only, but resulted in an even higher OR of $9.8(95 \%$ CI $2 \cdot 6-36 \cdot 6, P=0 \cdot 01)$. Conditional logistic regression of the matched pairs (exactly as in the Finnish study) yielded again an unchanged OR of $9 \cdot 1(95 \% \mathrm{CI}$ $2 \cdot 6-31 \cdot 2)$ of yet higher significance $(P=0 \cdot 0004)$, second only to the risk factor wood-cutting, with OR $15 \cdot 5(95 \%$ CI $2 \cdot 0-119 \cdot 6, P=0 \cdot 008)$ [4].

These results support the intriguing Finnish hypothesis that the condition of the human airways, altered by chronic smoking, might facilitate the uptake of aerosolized hantaviruses. If so, then the mean number of smoked cigarettes per day should give an even better indication, a factor not examined, however, in this study. In contrast, the Belgian study showed that NE cases smoked significantly more than controls [4].

'Type of housing' and 'living environment' were described in the Finnish study as having strong correlations, as expected. The dominant biotope in Finland is the boreal coniferous forest or taiga, in contrast to the deciduous broad-leaf forests in Western Europe. However, the PUUV reservoir in both regions is a rodent from the family Cricetidae, the bank vole or Myodes glareolus, a common small mammal in both forest biotopes [5]. So, the strongest risk factor linked to housing and/or living environment might be in fact the distance of the human habitat or working place to the forest, an element 
again not examined in the study of Vapalahti et al. Indeed, living $<50 \mathrm{~m}$ from the forest emerged as a risk factor for NE (adjusted OR 1.9, $95 \%$ CI 0.4-9.2) in another early Franco-Belgian case-control study, not mentioned in the Finnish study, probably because the $P$ value was only $0 \cdot 43$ [6]. Interestingly, careful analysis of a recent so-called urban NE outbreak in 'metropolitan' Cologne (Western Germany), teaches us that most patients lived, worked, or recreated near or in the semi-forested outer city park, significantly enough called 'Stadtwald' (forest of the city) [7].

Finally, the second most important risk factor for NE found in the Finnish study was 'living in buildings with holes allowing rodents to enter' [1]. Logically, the opposite, i.e. living in houses correctly sealed off for rodents should have a protective effect, resulting in a negative risk factor. Such a negative risk factor, inherent to 'rodent control at home', had already been reported in the 1999 Franco-Belgian case-control study (adjusted OR 0·3, $95 \%$ CI 0·1-1·1) [6], but was esteemed in the Finnish study as 'non-significant', rather than borderline significant with a $P$ value of 0.06 .

'Rodent control at home', or blocking the entry of rodents in human dwellings, is by far the most effective, least dangerous, and least expensive way of continuous indoor prevention of NE, as the Finnish authors propose in their conclusions. Had they used the opposite inquiry in their questionnaire (i.e. absence instead of presence of 'holes in the building'), they almost certainly would have reached either the same or an even more convincing result than their Franco-Belgian colleagues 11 years before.

\section{Declaration of Interest}

None.

\section{References}

1. Vapalahti K, et al. Case-control study on Puumala virus infection: smoking is a risk factor. Epidemiology and Infection 2010; 138: 576-584.

2. Clement J, Colson P, McKenna P. Hantavirus pulmonary syndrome in New England and Europe. New England Journal of Medicine 1994; 331: 545-546.

3. Clement $\mathbf{J}$, et al. Hantaviruses: underestimated respiratory viruses? Clinical Infectious Diseases 2008; 46: $477-$ 479.

4. Van Loock F, et al. A case-control study after a hantavirus outbreak in the South of Belgium: who is at risk? Clinical Infectious Diseases 1999; 28: 834-839.
5. Clement $\mathbf{J}$, et al. Beechnuts and outbreaks of nephropathia epidemica (NE): of mast, mice and men. Nephrology Dialysis Transplantation 2010; 25: 17401746.

6. Crowcroft NS, et al. Risk factors for human hantavirus infection: Franco-Belgian collaborative case-control study during the 1995-6 epidemic. British Medical Journal 1999; 318: 1737-1738.

7. Essbauer SS, et al. Nephropathia epidemica in metropolitan area, Germany. Emerging Infectious Diseases 2007; 13: 1271-1273.

J. P. CLEMENT ${ }^{1}$, N. S. CROWCROFT ${ }^{2}$, P. MAES ${ }^{1}$,

F. VAN LOOCK ${ }^{3}$, P. $\operatorname{COLSON}^{4}$ AND M. VAN RANST ${ }^{1}$

${ }^{1}$ Hantavirus Reference Centre, Laboratory of

Clinical Virology, Rega Institute, University of Leuven,

Belgium

${ }^{2}$ Ontario Agency for Health Protection and Promotion, Ontario, Canada

${ }^{3}$ Health Threat Unit, DG-SANCO/C3 HITEC,

Luxembourg

${ }^{4}$ Internal Medicine, Centre Hospitalier de Jolimont-Lobbes, Lobbes, Belgium

(Email: jan.clement.dr@telenet.be)

\section{The authors reply:}

We thank Dr Clement and colleagues for their comments and interest in our report [1] and for raising important points concerning risks for avoidance of hantavirus infections. We regret that we did not discuss their results concerning similar effects of smoking - as well as other risk factors - found in the previous Belgian study [2], especially as these results corroborate our findings. Smoking was indeed already found as a significant 'host factor' in this first European hantavirus case-control study. However, it was not mentioned among the three risk factors identified in their multivariate analysis; the authors did not discuss smoking in their discussion; nor did they mention it in their abstract. We focused on effects of smoking by separately studying, for example, 'virtual' controls with 'average smoking habits' and tried to ensure that this effect was not due to any confounding factors.

We also agree with Clement and colleagues that 'rodent control at home' is one of the useful things one can do to prevent hantavirus infection during outbreaks. It is quite common after tabulating and analysing questionnaire data that one has late second thoughts of how questions should have been formulated. However, our findings also suggest that using poison could be safer than using traps, which potentially exposes persons to hantavirus 
contamination from (dead) rodents. The case-control studies done so far should give information at least to focus future epidemiological studies and to perhaps review some recommendations on how to prevent hantavirus infections. Prevention is a priority as long as there is no vaccine available for this rodent-borne infection.

\section{References}

1. Vapalahti K, et al. Case-control study on Puumala virus infection: smoking is a risk factor. Epidemiology and Infection 2010; 138: 576-584.
2. Van Loock F, et al. A case-control study after a hantavirus outbreak in the South of Belgium: who is at risk? Clinical Infectious Diseases 1999; 28: 834-839.

K. VAPALAHTI ${ }^{1,2}$, A. - M. VIRTALA ${ }^{3}$, A. VAHERI ${ }^{1,2}$ AND O. VAPALAHTI ${ }^{1,2,3}$

${ }^{1}$ Department of Virology, Haartman Institute, University of Helsinki, Helsinki, Finland

${ }^{2}$ Department of Virology, Helsinki University Hospital Laboratory, Helsinki, Finland

${ }^{3}$ Department of Veterinary Biosciences, Faculty of Veterinary Medicine, University of Helsinki, Helsinki, Finland

(Email: olli.vapalahti@helsinki.fi) 